\title{
Hesitant Fuzzy Worth: an innovative ranking methodology for hesitant fuzzy subsets
}

\author{
J. C. R. Alcantud ${ }^{a, *}$, R. de Andrés Calle ${ }^{a}$, María J. M. Torrecillas ${ }^{b}$ \\ ${ }^{a}$ Facultad de Economía y Empresa and Multidisciplinary Institute of Enterprise (IME) \\ Universidad de Salamanca, 37007 Salamanca, Spain \\ ${ }^{b}$ Department of Economics and Business, University of Almería, 04120 Almería, Spain
}

\begin{abstract}
We introduce a novel methodology for ranking hesitant fuzzy sets. It builds on a recent, theoretically sound contribution in Social Choice. In order to justify the applicability of such analysis, we develop two real implementations: ( $i$ ) new metarankings of world academic institutions that build on real data from three reputed agencies, and $(i i)$ a new procedure for improving teaching performance assessments which we illustrate with real data collected by ourselves. These applications provide new grounds for the theoretical model by hesitant fuzzy sets.
\end{abstract}

Keywords: Multi-attribute decision making; Hesitant fuzzy element; Hesitant fuzzy set; Ranking function; Score; Hesitant fuzzy worth; Metaranking of universities; Teaching performance assessments.

\section{Introduction}

It is well-known that the analysis of many real-world situations is intricate and controversial since it is affected by human behavior and its subjectivity. Problems defined under uncertain conditions can be approached from different positions. For example, Computing with Words can be used for reasoning and computing with perceptions. This is useful in the cases involving people that express their preferences or the potential of each alternative through qualitative rather than quantitative values (cf., [1]). When the researcher has

\footnotetext{
${ }^{*}$ Corresponding author

Email addresses: jcr@usal.es (J. C. R. Alcantud), rocioac@usal.es (R. de Andrés Calle), mjmtorre@ual.es (María J. M. Torrecillas) 
data in the form of measurements, besides standard optimization procedures she can refer to Fuzzy Set Theory. This literature has provided useful tools for the management of such inconveniences in decision-making contexts (see [2], [3] and [4] as a sample; [5] is an extensive analysis of papers about fuzzy multi-criteria decision making published in the period 1994-2014). However it has long been recognized that in some practical problems, imprecise individual or group knowledge cannot be suitably represented by fuzzy sets (FSs) while some generalizations of this notion provide better insights.

To be precise, [6] introduced a new extension of fuzzy sets called hesitant fuzzy sets (HFSs) that is motivated by such limitations. HFSs incorporate many-valued sets of memberships (hesitant fuzzy elements, HFEs defined in [7]), which permits to manage simultaneous sources of vagueness.

The motivation for using this concept in decision making is clearly explained e.g., in $\mathrm{Xu}$ [8]. This reference justifies that hesitant fuzzy elements and sets have produced an extensive theoretical and applied literature. In the recent authoritative survey of HFSs [9], the authors summarize many useful and valuable decision making methods to solve hesitant fuzzy multicriteria decision making problems and propose further applications of HFSs to decision making. Within the extended field of Hesitancy in Fuzzy Sets, application papers that contribute to multicriteria decision making include [7], [10], [11], [12], [13], [14], [15] and [16]. Multiexpert multicriteria decision making under this requirement has been explored by Xia et al. [17] or Tan et al. [18] among others. Rodríguez et al. [9], Section 7, emphasize that "a new trend is the application of the theoretical models to real problems". In this paper we adhere to their position. We introduce a new theoretical procedure for ranking HFSs, and then we apply the model to a real situation, to wit, the elaboration of a metaranking of world academic institutions.

The solutions to the problem of prioritizing HFSs on a fixed set of alternatives rely on two basic methodologies which in some sense, perform similar operations in different order. Xia and $\mathrm{Xu}$ [7], Section 4, propose to aggregate HFEs into a single HFE and then rank these constructs according to an indicator or score. Farhadinia [19] attempts to act directly on the HFSs by computing the scores of their constituting HFEs, which are subsequently aggregated into an indicator. $\mathrm{Xu}$ [8] contains an extensive discussion of other works along these lines.

Here we propose a new general approach that complements the aforementioned particular solutions. In order to formalize these procedures for prioritizing HFSs we use the novel abstract concept of ranking function on 
hesitant fuzzy sets. This notion attaches a numerical 'desirability' indicator with each HFS on the set of alternatives. Ranking functions appear to be the most natural way to prioritize HFSs, in line with earlier uses of specific ranking functions on FSs (e.g., [20, 21, 22]).

In our proposal the suitable application of scores on HFEs (specifically from [7], [10], and [19], who introduces a Geometric score, see also [23]) is complemented with a novel construction in this framework. It was imported from Social Choice -cf., the worth in Herrero [24]- to fuzzy soft set based decision making in [25]. There it was announced that the same tool could be successfully implemented in other related soft computing models, which we accomplish here for the analysis of HFSs. The conjunction of these two steps (score followed by application of the adapted notion of worth to the fuzzy setting) gives rise to a Hesitant Fuzzy Worth which naturally induces a ranking of the alternatives.

We also report on the results of two real and original applications that illustrate our theoretical contribution and at the same time, further validate the model by HFSs. In the first case we build on real data from three reputed rankings of world universities, namely, the Academic Ranking of World Universities (also known as Shanghai Ranking), the QS World University Rankings and the Times Higher Education World University Rankings. We justify that this multi-source imprecise information naturally fits the model by HFSs, thus providing additional truthful justification of the need for such concept. Then we benefit from this modelization in order to produce metarankings of academic institutions that result from the application of our methodology for ranking HFSs. A comparison with existing methodologies is performed too. Then in the second case we use our methodology to rank teachers' performance according to the results of their students' surveys. We rely on anonymized real data from student evaluations of staff of the department of Economics and Economic History, Universidad de Salamanca, Spain. We only present a fraction of the data to preserve confidentiality. But our procedure can be used to design a universal teacher performance assessment instrument too. This possible target is beyond the scope of this paper.

This paper is organized as follows. Section 2 establishes some basic notation and definitions related to hesitant fuzzy sets. In addition a review of the literature related to hesitant multi-attribute decision making problems is included. Section 3 contains our theoretical analysis of the general notion of ranking function for HFSs and the construction of the Hesitant Fuzzy Worth 
associated with any hesitant fuzzy decision matrix. Section 4 contains two practical examples that illustrate the use of the Hesitant Fuzzy Worth in decision making contexts. In Section 5 we develop our first real application of techniques for the hierarchization of world universities characterized by hesitant fuzzy sets. Then we compare our results with the rankings obtained by the application of known methodologies. In Section 6 we explain how our novel procedure permits to give a ranking of teachers by their students' surveys, using real data from a fraction of department's staff. We conclude in Section 7.

\section{Notation, definitions and review of the literature}

In this Section we describe basic definitions and notions of hesitant fuzzy set. In addition, we review the traditional methodologies used to rank HFSs in multi-attribute decision making contexts.

\subsection{Hesitant fuzzy elements and hesitant fuzzy sets}

For any set $A, \mathcal{P}^{*}(A)$ denotes the set of non-empty subsets of $A$, and $\mathcal{F}^{*}(A)$ denotes the set of non-empty finite subsets of $A$.

Definition 1 (Xia and $\mathrm{Xu}[7]$ ). A hesitant fuzzy element (HFE) is a nonempty, finite subset of $[0,1]$. The set of HFEs is denoted by $\mathcal{F}^{*}([0,1])$.

Generic HFEs are expressed as $h=\left\{h^{1}, \ldots, h^{l_{h}}\right\}$, where $h^{1}<\ldots<h^{l_{h}}$ and $l_{h}=|h|$ is the cardinality of the HFE $h$. In particular, $h=\{1\}$ is usually called the full HFE, and $h=\{0\}$ is usually called the empty HFE. [13] made some deep clarifications on these special HFEs.

Henceforth we refer to $X$, a fixed set of alternatives.

Definition 2 (Torra [6]). A hesitant fuzzy set (HFS) on $X$ is a function from $X$ to $\mathcal{P}^{*}([0,1])$. A typical hesitant fuzzy set on $X$ is a function from $X$ to $\mathcal{F}^{*}([0,1])$. The set of typical HFSs on $X$ is denoted by $\operatorname{HFS}(X)$, and $\operatorname{HFS}(X)$ means the set of HFSs on $X$. Unless otherwise stated, HFSs are assumed to be typical.

From a formal point of view, a (typical) HFS is a subset $M \subseteq X \times \mathcal{F}^{*}([0,1])$ such that for each $x \in X$, there is exactly one element $h_{M}(x) \in \mathcal{F}^{*}([0,1])$ such that $\left(x, h_{M}(x)\right) \in M$. Put differently, typical HFSs on $X$ associate an HFE $h_{M}(x)$ with each element $x \in X$. 
Every HFS on $X$ defines a set of membership values for each element of $X$, and in the case that the HFS is typical such set is always finite. HFEs represent the set of possible membership values of a typical hesitant fuzzy set at an alternative.

By restricting ourselves to $\mathcal{F}^{*}([0,1])$, i.e., non-empty HFEs, we disregard 'nonsense elements' in each HFS: on each alternative, at least one assessment must be made.

From a practical point of view, the hesitant fuzzy set $M$ can be represented as $M=\left\{\left(x, h_{M}(x)\right) \mid x \in X\right\}$. For example, following [6] we define $M^{*}=\{(x,\{1\}) \mid x \in X\}$ as the ideal or full HFS on $X$, and $M^{-}=\{(x,\{0\}) \mid x \in X\}$ as the anti-ideal or empty HFS on $X$.

Clearly, when all HFEs involved in the definition of an HFS on $X$ are singletons we can identify such HFS with a fuzzy set (FS) on $X$. That is to say, HFSs of the form $M=\left\{\left(x, h_{M}(x)\right) \mid x \in X, h_{M}(x)=\left\{M_{x}\right\}\right\}$ can be identified with the FS on $X$ whose membership function is $\mu_{M}: X \longrightarrow[0,1]$ such that $\mu_{M}(x)=M_{x}$. The ideal and anti-ideal HFS on $X$ are examples of such structural duality. Henceforth $\mathbf{F S}(X)$ denotes the fuzzy subsets of $X$, and we can embed $\mathbf{F S}(X)$ into $\operatorname{HFS}(X)$ through the aforementioned identification.

In agreement with our notation for HFEs, for each typical hesitant fuzzy set $M$ on $X$ we write $h_{M}(x)=\left\{h_{M}^{1}(x), \ldots, h_{M}^{l_{M}(x)}(x)\right\}$ with the property that $h_{M}^{1}(x)<\ldots<h_{M}^{l_{M}(x)}(x)$, and $l_{M}(x)=\left|h_{M}(x)\right|$ is the cardinality of the HFE $h_{M}(x)$. Observe that if the set of membership values at an element is not finite (i.e., if we refer to a non-typical HFS) then such arrangement in increasing order cannot be made in general. In any case, because $h_{M}(x)$ is a set, repetitions are excluded by definition.

\subsection{Methodologies for ranking hesitant fuzzy sets}

In the context of decision making, when the opinions are collected according to either the model by HFEs (one single attribute) or by HFSs (multiple attributes), the decision maker needs to reach a decision that considers all that information (see Figure 1). Farhadinia [19] explains that hesitant fuzzy multi-attribute decision making is a trendy research area, and that in this context, ranking of alternatives plays a crucial role.

We devote this Subsection to review different existing methodologies that rank HFSs. We focus our attention on decision making problems where the evaluations of alternatives are cardinal. Therefore, we avoid ranking 
approaches consistent with ordinal data such as ELECTRE [26], QUALIFEX [27], TODIM [16], TOPSIS [28], VIKOR [29] and so forth. We classify the existing methodologies in two groups: methods based on scores for HFEs, and methods based on distances to ideal HFSs.

We proceed to explain the details of both alternatives.

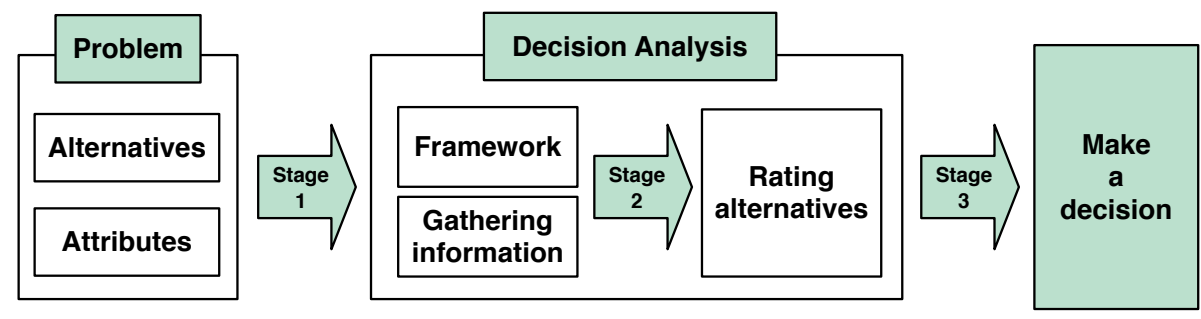

Figure 1: Scheme of a decision making problem. Stage 2 is reviewed in Subsection 2.2 when the problem consists of hesitant multi-attribute information.

\subsubsection{Ranking methodologies based on HFEs scores}

Scores on HFEs permit to rank HFEs in an immediate manner: the higher the score of an HFE, the better it is considered. Nevertheless the formal concept of score on HFEs remains undefined as far as we are aware of. Consequently, we first introduce this formal concept in Definition 3 below:

Definition 3. A score on HFEs is a mapping $s: \mathcal{F}^{*}([0,1]) \longrightarrow[0,1]$ such that $s(h)=1$ if and only if $h=\{1\}$.

Similar in spirit is Farhadinia [19, Section 2] who defines HFE score functions for HFEs with the same number of (possibly repeated) elements only. Besides this restriction that we eschew, his limited definition imposes some unnecessary boundary conditions.

Now we proceed to recall some traditional scores on typical HFEs. ${ }^{1}$

\footnotetext{
${ }^{1}$ To further clarify the misleading use of the term "score" in the literature, it must be mentioned that various authors have used the common term "the score function for HFEs" to designate one of these single specific examples, rather than a generic notion with its own meaning. This is clearly explained in the up-to-date survey [9].
} 
Definition 4 (Xia and $\mathrm{Xu}[7]$ ). The Xia and $\mathrm{Xu}$ score on HFEs is the mapping $\mathcal{S}_{1}: \mathcal{F}^{*}([0,1]) \longrightarrow[0,1]$ such that

$$
\mathcal{S}_{1}(h)=\frac{h^{1}+\ldots+h^{l_{h}}}{l_{h}} .
$$

Definition 5 (Farhadinia [10]). Consider a monotone non-decreasing sequence $\{\delta(1), \ldots, \delta(n), \ldots\}$ of positive numbers. The Farhadinia score on HFEs is the mapping $\mathcal{S}_{2}: \mathcal{F}^{*}([0,1]) \longrightarrow[0,1]$ such that

$$
\mathcal{S}_{2}(h)=\frac{\delta(1) h^{1}+\ldots+\delta\left(l_{h}\right) h^{l_{h}}}{\delta(1)+\ldots+\delta\left(l_{h}\right)} .
$$

This includes $\mathcal{S}_{1}$ when the sequence is $\{1,1, \ldots, 1, \ldots\}$.

Definition 6 (Farhadinia [19]). The geometric-mean score function on HFEs is the mapping

$$
\mathcal{S}_{G M}(h)=\left(\prod_{i=1}^{l_{h}} h^{i}\right)^{\frac{1}{l_{h}}} .
$$

The following example illustrates some of the HFEs scores described above.

Example 1. Let $X=\left\{x_{1}, x_{2}\right\}$. Consider the typical HFS on $X$ defined by

$$
\begin{aligned}
& M: X \longrightarrow \mathcal{F}^{*}([0,1]) \\
& x_{1} \quad h_{M}\left(x_{1}\right)=\{0.55,0.7\} \\
& x_{2} \quad h_{M}\left(x_{2}\right)=\{0.75,0.8,0.85\}
\end{aligned}
$$

The scores associated with $x_{1}$ and $x_{2}$ are:

- With respect to $\mathcal{S}_{1}$ :

$$
\begin{aligned}
& \mathcal{S}_{1}\left(h_{M}\left(x_{1}\right)\right)=\frac{0.55+0.7}{2}=\frac{5}{8}=0.625 \\
& \mathcal{S}_{1}\left(h_{M}\left(x_{2}\right)\right)=\frac{0.75+0.8+0.85}{3}=\frac{4}{5}=0.8
\end{aligned}
$$


- With respect to $\mathcal{S}_{2}$ :

$$
\begin{aligned}
& \mathcal{S}_{2}\left(h_{M}\left(x_{1}\right)\right)=\frac{0.55+2 \cdot 0.7}{1+2}=\frac{13}{20}=0.65 \\
& \mathcal{S}_{2}\left(h_{M}\left(x_{2}\right)\right)=\frac{0.75+2 \cdot 0.8+3 \cdot 0.85}{1+2+3}=\frac{49}{60} \approx 0.82
\end{aligned}
$$

- With respect to $\mathcal{S}_{G M}$ :

$$
\begin{aligned}
& \mathcal{S}_{G M}\left(h_{M}\left(x_{1}\right)\right)=(0.55 \cdot 0.7)^{\frac{1}{2}} \approx 0.620 \\
& \mathcal{S}_{G M}\left(h_{M}\left(x_{2}\right)\right)=(0.75 \cdot 0.8 \cdot 0.85)^{\frac{1}{3}} \approx 0.798
\end{aligned}
$$

The aforementioned notions permit to produce solutions in the model by hesitant fuzzy single-attribute information (formally, options are captured by HFEs). When options are captured by HFSs we refer to the general model by hesitant fuzzy multi-attribute information, and we need an intermediate step that characterizes each alternative by a HFE (see Figure 1, Stage 2).

Xia and $\mathrm{Xu}$ [7] fill that step by introducing a number of hesitant fuzzy aggregation operators that transform HFSs into HFEs, a successful approach subsequently followed by other authors (cf., Table 1). In short, Xia and Xu's methodology proceeds by first associating each alternative with a HFE, and then ranking the resultant HFEs by the appeal to a score.

Recently, Farhadinia [19] proposes a series of score functions for HFSs based on known score functions on HFEs. With this tool he develops a HFS score-based method to solve multi-attribute decision making problems with hesitant information (cf., Table 1). Put shortly, his proposal proceeds in two phases: firstly an score function is applied to each HFE, and then a similar notion computes the final value (cf., [19, Definition 4.1 and page 107]).

In order to compare our new procedure with existing methodologies below in Section 5, we select the successful approaches proposed in [7] and [19].

\subsubsection{Ranking methodologies based on distance measures to reference ele- ments}

The general idea that 'distances' to distinguished items are useful indicators of the ordering of the alternatives has been developed from multiple approaches. The basic idea consists of setting up a reference set(s) and then all the alternatives are compared with the reference set(s). Following this 
postulate, one can rank HFSs according to their distance or similarity to adequate reference elements.

The distance measures between hesitant fuzzy elements (HFEs) and hesitant fuzzy sets (HFSs) are introduced in the literature by Xu and Xia [12, 30]. Li et al. [31] propose some new distance and similarity measures on hesitant fuzzy sets. They apply them to various multi-criteria decision making problems.

Definition 7 (Xu and Xia [30]). A distance measure between HFSs on $X$ is a function $d: \mathbf{H F S}(X) \times \mathbf{H F S}(X) \longrightarrow[0,1]$ that satisfies: for every $M, N \in \operatorname{HFS}(X)$,

(d.1) $d(M, N)=0$ if and only if $M=N$;

(d.2) $d(M, N)=d(N, M)$.

When $d$ is a distance measure between HFSs on $X, s=1-d$ is a similarity measure between HFSs on $X$ (Xu and Xia [30, Def. 3]) and conversely. Observe that in this case, $s(M, N)=1$ if and only if $M=N$.

Let us fix a distance measure $d$ between HFSs on $X$. Then for every $M, M^{\prime} \in \operatorname{HFS}(X)$ one can $\operatorname{rank} M$ above $M^{\prime}$ if and only if $d^{*}(M) \leqslant d^{*}\left(M^{\prime}\right)$, where $d^{*}(M)=d\left(M, M^{*}\right)$ is the distance to the ideal HFS. This captures the idea that the closer to the ideal HFS, the better.

Or alternatively, for every $M, M^{\prime} \in \mathbf{H F S}(X)$ one can decide to rank $M$ above $M^{\prime}$ if and only if $d^{-}(M) \geqslant d^{-}\left(M^{\prime}\right)$, where $d^{-}(M)=d\left(M, M^{-}\right)$is the distance to the anti-ideal HFS. This captures the idea that the farther away from the anti-ideal HFS, the better. We eschew the corresponding analysis based on similarity measures, which derives easily from the simple transformation between similarity and distance measures.

In order to apply this ranking procedure, the researcher can take advantage of [9, Subsection 5.1] to fix a suitable distance between HFSs from the literature. In particular, one can utilize prominent distances from [30]: the hesitant weighted Hamming distance (HWHD), the hesitant weighted Euclidean distance (HWED), the hesitant weighted Hamming-Hausdorff distance (HWHHD), the hesitant weighted Euclidean-Hausdorff distance (HWEHD), et cetera. In this paper we focus on these distance measures between HFSs proposed in [30]. Further proposals of distances and similarity measures can be found e.g., in [31] (cf., Table 1). 
To conclude, Table 1 summarizes and classifies the methodologies that we have reviewed.

\begin{tabular}{|c|c|}
\hline Author(s) & $\operatorname{Tool}(\mathrm{s}) / \operatorname{method}(\mathrm{s})$ \\
\hline & Based on HFEs scores \\
\hline [7] & $\begin{array}{l}\text { Generalized hesitant fuzzy weighted averaging operator (GHFWA) } \\
\text { Generalized hesitant fuzzy weighted geometric operator (GHFWG) }\end{array}$ \\
\hline$[19]$ & Series of score functions for hesitant fuzzy sets \\
\hline$[32]$ & $\begin{array}{l}\text { Weighted hesitant fuzzy geometric Bonferroni mean (WHFGBM) } \\
\text { Weighted hesitant fuzzy Choquet geometric Bonferroni mean (WHFCGBM) }\end{array}$ \\
\hline [33] & Hesitant fuzzy prioritized operators \\
\hline & Based on distance measures \\
\hline$[12]$ & Distance measures of hesitant fuzzy elements \\
\hline [30] & Generalized hesitant weighted distance \\
\hline [31] & Distance and similarity measures considering hesitancy degree \\
\hline
\end{tabular}

Table 1: Summary table of studies related to ranking HFSs

\section{A novel ranking function for hesitant fuzzy subsets}

We devote our theoretical analysis to the concept of a ranking function for HFSs on a set $X$, which permits to attach numbers with each HFS on $X$. This in turn permits to induce an ordering on $\operatorname{HFS}(X)$ which prioritizes the projects or alternatives characterized by them, among other possible uses. Of course the same is true for the case of typical HFSs. Formally we have the following novel definition:

Definition 8. A ranking function on $\boldsymbol{H F S}(X)$ is a mapping

$$
F: \boldsymbol{H F S}(X) \longrightarrow[0,+\infty)
$$

Similarly we define ranking functions on $\operatorname{HFS}(X)$ or even $\boldsymbol{F S}(X)$ by successively restricting the domain.

Now we take advantage of a recent advancement in Social Choice that coupled with the use of scores, provides a completely redesigned approach to ranking hesitant fuzzy sets. We first give an informal briefing on its various 
steps. Afterwards we present the formal construction of the algorithm and an illustrative example.

The procedure starts with some complete preorders. In our case we compute the scores associated with each alternative and attribute, and then each of the attributes produces one prioritization of the alternatives according to their successive scores. Now with the complete preorders defined by these prioritizations we proceed to rank the alternatives according to the endogenous scoring rule put forward in [24]. To this purpose we construct a positive matrix whose cells are defined as Borda counts in its diagonal, and Condorcet dominations off its diagonal (in all cases with respect to the aforementioned scores). And the unique normalized eigenvector associated with its dominant eigenvalue provides the ranking.

The precise definition that captures this construction is as follows.

There are $k$ objects $o_{1}, \ldots, o_{k}$, and the set $X$ of characteristics that qualify them has $q$ components. Each object is identified with a typical HFS on $X$. Therefore our input is a Hesitant Fuzzy Decision Table with $k$ rows and $q$ columns. Each cell $(i, j)$ contains the corresponding HFE that describes object $i$ according to characteristic $j$.

Let us fix a score on HFEs (cf., Definition 3). By applying such predefined score to the constituent HFEs of our Hesitant Fuzzy Decision Table, a new $k \times q$ score table is produced. Its cell $(i, j)$ is denoted by $t_{i j}$. It contains the score attached to the $j$-th characteristic of object $i$.

Now we construct a new $k \times k$ matrix $C=\left(c_{i j}\right)_{k \times k}$ according to the following rules:

(a) When $i \neq j, c_{i j}$ is the number of characteristics for which the score attached to $o_{i}$ is strictly greater than the score attached to $o_{j}$. Thus $c_{i j}$ is the number of characteristics $m$ for which $t_{i m}-t_{j m}>0$, or the number of positive values in the finite sequence $t_{i 1}-t_{j 1}, t_{i 2}-t_{j 2}, \ldots$

(b) For each $i$, let $m_{i}=\sum_{j \neq i} c_{j i}$ be the sum of the non-diagonal elements in column $i$ of $C$ and then define $c_{i i}=q(k-1)-m_{i}$.

Herrero [24] carefully argues that the tallies in this construction capture the notions that we explain above. This is to say, our matrix $C$ contains Borda counts in its diagonal, and Condorcet dominations off its diagonal. These are two focal notions in group decision making from the viewpoint of Social Choice. For details on this extraneous issue, the reader is addressed to $[24]$. 
Because the square matrix $C$ has positive entries, the Perron-Frobenius theorem asserts that $C$ has a unique largest real eigenvalue, and also that its corresponding eigenvector can be chosen to have strictly positive components.

Herrero proves that the Perron-Frobenius eigenvalue of $C$ is forcefully $q(k-1)$. In order to prioritize the objects we compute a unique normalized eigenvector $\mathcal{H}=\left(\mathcal{H}_{1}, \cdots, \mathcal{H}_{k}\right)$ associated with it. Normalization is performed so that $\mathcal{H}_{1}+\cdots+\mathcal{H}_{k}=1$.

Finally we arrange the objects from highest to smallest component. To capture these important amounts we formalize the following concept:

Definition 9. For a fixed score on HFEs, $\mathcal{H}_{i}$ is called the Hesitant Fuzzy Worth $(H F W)$ associated with object $o_{i}$.

In conclusion, our procedure grades the objects according to their Hesitant Fuzzy Worth. This is the corresponding component of a normalized eigenvector associated with the dominant eigenvalue of a matrix defined through scores. The higher its HFW, the better an alternative is. The investigation by Herrero permits to import relevant features of this solution, like the exact identification of the dominant eigenvalue of the matrix directly from the size of the corresponding score table.

\section{Practical examples}

Prior to our real and original applications, in this Section we illustrate our ranking procedure with two brief case studies. The first one is an artificial example that shows the various steps in our procedure. The second one uses data from a real situation in order to produce a ranking of domestic airlines by their service quality.

\subsection{An artificial practical example}

In this illustrative example we consider the problem characterized by the Hesitant Fuzzy Decision Table given in Table 2. Suppose that we are bound by $\mathrm{Xu}$ and Xia's score. We proceed to apply our proposed ranking approach in order to prioritize the objects of this artificial problem.

This problem consists of $k=3$ objects and $q=3$ characteristics. It addresses to the score table given in Table 3 , from which it is simple to 
compute the $k \times k$ matrix

$$
C=\left(\begin{array}{lll}
2 & 1 & 1 \\
2 & 5 & 2 \\
2 & 0 & 3
\end{array}\right)
$$

The dominant eigenvalue of $C$ is $6=3 \cdot(3-1)$. A normalized eigenvector associated with this eigenvalue is $\left(\frac{1}{5}, \frac{2}{3}, \frac{2}{15}\right)$, which is computed from the system of linear equations $C \vec{x}=6 \vec{x}$ and the normalization rule. Therefore $\mathcal{H}_{1}=0.2, \mathcal{H}_{2} \approx 0.67, \mathcal{H}_{3} \approx 0.13$ are the respective Hesitant Fuzzy Worths associated with the objects, and our algorithm suggests that $\mathrm{O}_{2}$ should be selected.

Table 2: A representation of the HFSs for the artificial case study in subsection 4.1.

\begin{tabular}{llll} 
Options & Characteristic 1 & Characteristic 2 & Characteristic 3 \\
\hline$o_{1}$ & $(0.30,0.50)$ & $(0.60,0.65,0.70)$ & $(0.70,0.80,0.90)$ \\
$o_{2}$ & $(0.65,0.75)$ & $(0.90,0.95,1)$ & $(0.65,0.85)$ \\
$o_{3}$ & $(0.60,0.80)$ & $(0.70,0.80)$ & $(0.50,0.60,0.70)$ \\
\hline
\end{tabular}

Table 3: Score table associated with the case study in subsection 4.1. Xia and Xu's score is adopted.

\begin{tabular}{llll} 
Options & Characteristic 1 & Characteristic 2 & Characteristic 3 \\
\hline$o_{1}$ & 0.40 & 0.65 & 0.80 \\
$O_{2}$ & 0.70 & 0.95 & 0.75 \\
$o_{3}$ & 0.70 & 0.75 & 0.60 \\
\hline
\end{tabular}

\subsection{Practical application: a ranking of domestic airlines by service quality}

In this subsection we illustrate our ranking procedure with a brief example that uses real data from Liou et al. [34], as cited in Liao and Xu [13]. The latter authors explain that Liou et al. [34] conducted a survey about passengers' importance and perception of quality of service in four domestic airlines, according to which they produce a classification that relies on hesitant information regarding four major criteria. We collect that information in Table 4. 
Table 4: Hesitant fuzzy decision matrix for the application in subsection 4.2 (cf., [13, Table 1]).

\begin{tabular}{lllll} 
Airlines & Criterion 1 & Criterion 2 & Criterion 3 & Criterion 4 \\
\hline UNI Air & $(0.6,0.7,0.9)$ & $(0.6,0.8)$ & $(0.3,0.6,0.9)$ & $(0.4,0.5,0.9)$ \\
Transasia & $(0.7,0.8,0.9)$ & $(0.5,0.8,0.9)$ & $(0.4,0.8)$ & $(0.5,0.6,0.7)$ \\
Mandarin & $(0.5,0.6,0.8)$ & $(0.6,0.7,0.9)$ & $(0.3,0.5,0.7)$ & $(0.5,0.7)$ \\
Daily Air & $(0.6,0.9)$ & $(0.7,0.9)$ & $(0.2,0.4,0.7)$ & $(0.4,0.5)$ \\
\hline
\end{tabular}

The relevant criteria in Table 4 concern (1) Booking and ticketing service, (2) Checking and boarding process, (3) Cabin service, and (4) Responsiveness. When we use Xia and Xu's score we obtain Table 5 (cf., [13, Table 2]).

Table 5: Score table associated with the case study in subsection 4.2 (cf., [13, Table 1]). Xia and Xu's score is adopted.

\begin{tabular}{lllll} 
Airlines & Criterion 1 & Criterion 2 & Criterion 3 & Criterion 4 \\
\hline UNI Air & 0.7333 & 0.7 & 0.6 & 0.6 \\
Transasia & 0.8 & 0.7333 & 0.6 & 0.6 \\
Mandarin & 0.6333 & 0.7333 & 0.5 & 0.6 \\
Daily Air & 0.75 & 0.8 & 0.4333 & 0.45 \\
\hline
\end{tabular}

Following our procedure we need to produce the following $4 \times 4$ matrix:

$$
C=\left(\begin{array}{cccc}
7 & 0 & 2 & 2 \\
2 & 11 & 2 & 3 \\
1 & 0 & 6 & 2 \\
2 & 1 & 2 & 5
\end{array}\right)
$$

The dominant eigenvalue of $C$ is $12=4 \cdot(4-1)$. A normalized eigenvector associated with this eigenvalue is $(0.0816327,0.714286,0.0612245,0.142857)$, which is computed from the system of linear equations $C \vec{x}=12 \vec{x}$ and the normalization rule. Therefore $\mathcal{H}_{1} \approx 0.08, \mathcal{H}_{2} \approx 0.71, \mathcal{H}_{3} \approx 0.06, \mathcal{H}_{4} \approx 0.14$ are the respective Hesitant Fuzzy Worths associated with the objects. Our algorithm suggests that Transasia has the better performance, which coincides with the conclusion in Liao and $\mathrm{Xu}[13]$. 


\section{Real application 1: academic metarankings of universities}

In this Section we exemplify the use of the aforementioned methodology for ranking a sample of seven of the most prestigious worldwide universities: Stanford, Harvard, Oxford, Cambridge, Berkeley, Princeton and Yale. We have refrained from making a lengthier study since the approach does not vary with the number of alternatives.

\subsection{Description of the real example}

There are various agencies that provide evaluations and rankings of higher education institutions, and typically they make finer evaluations on restricted broad fields or subjects like Social Sciences. This fits into our setting since we can regard this information as the evaluations by agents (the agencies conducting each survey) on different attributes (the fields) of the alternatives (the universities). It is not necessary that the agents are "good at evaluating all the attributes", therefore we accommodate to a request in [7]. For example, the 2014 Academic Ranking of World Universities (ARWU) does not evaluate the field Arts and Humanities which is evaluated by The Times Higher Education and the QS World University Rankings.

We use the evaluations of world universities rankings provided by three agencies (the experts), namely, the Academic Ranking of World Universities (Shanghai Ranking, henceforth Sh) by [35], the QS World University Rankings (henceforth QS) by [36] and Times Higher Education World University Rankings (henceforth THE) by [37]. We aggregate the hesitant fuzzy information that they convey in order to create new rankings for universities, as a function of the methodology that we adopt. These agencies have their own methodology to attach a score to each university, being 100 the maximum possible score. The experts are well known for their general world universities rankings, but they also offer university rankings by field. ${ }^{2}$ We establish five fields that are applicable to the three experts' classification procedures:

1. Arts and Humanities $(\mathrm{A} \& \mathrm{H})$.

2. Life Sciences and Medicine (L\&M).

3. Engineering and Technology (ENG).

4. Natural Sciences and Mathematics (SCI).

5. Social Sciences (SOC).

\footnotetext{
${ }^{2}$ Shanghai ranking uses the term "field", Times Higher Education "subject" and QS uses "faculty", referring to the same thing.
} 
For illustrative purposes we perform our analysis on the aforementioned 7 top universities. Table 6 gathers the original information, with the following provisos:

(a) The score attached to Sh and THE in the field "Life Sciences and Medicine" (L\&M) is the respective mean of their scores for the fields "Life Sciences" and "Medicine".

(b) Business Schools and Economics are included in the field "Social Sciences" (SOC). QS refers to this field as "Social Sciences and Management".

The raw information in Table 6 produces Table 7, which contains the resulting HFEs for each field and university. This is the problem's Hesitant Fuzzy Decision Table.

Table 6: Evaluations for the five fields made by the agencies (year 2014). A hyphen means 'no evaluation provided'.

\begin{tabular}{|c|c|c|c|c|c|c|c|c|c|c|c|c|c|c|}
\hline \multirow[b]{2}{*}{ University } & \multicolumn{2}{|c|}{$\mathbf{A} \& \mathbf{H}$} & \multicolumn{3}{|c|}{ L\&M } & \multicolumn{3}{|c|}{ ENG } & \multicolumn{3}{|c|}{ SCI } & \multicolumn{3}{|c|}{$\mathrm{SOC}$} \\
\hline & THE & QS & THE & Sh & QS & THE & Sh & $\overline{\text { QS }}$ & THE & Sh & $\overline{\text { QS }}$ & THE & Sh & QS \\
\hline Stanford University & 87.1 & 86.8 & 87.6 & 69.4 & 91.2 & 91.9 & 92.1 & 93.3 & 89.9 & 91.4 & 92.5 & 93.6 & 80.1 & 89.2 \\
\hline Harvard University & 86.1 & 89.7 & 91.3 & 100 & 98.2 & - & 65.1 & 85.7 & 90.2 & 100 & 92.3 & 91.9 & 100 & 96.3 \\
\hline University of Oxford & 84.4 & 99.1 & 91.1 & 60.9 & 92.3 & 87.6 & 64.4 & 86.1 & 87.3 & 72.3 & 90.4 & 93.5 & 59.9 & 94.2 \\
\hline University of Cambridge & 83.9 & 93.5 & 88.5 & 75.6 & 91.8 & 88.8 & 74.8 & 90.5 & 88.8 & 92.2 & 97.0 & 87.5 & 59.4 & 91.2 \\
\hline University of California, Berkeley & 81.4 & 87.2 & 81.6 & 58.0 & 85.6 & 90.6 & 86.8 & 90.2 & 89.9 & 96.3 & 93.4 & 86.9 & 79.6 & 87.3 \\
\hline Princeton University & 81.2 & 86.5 & 42.5 & 24.8 & 74.1 & 89.5 & 71.1 & 81.6 & 91.0 & 93.7 & 89.2 & 91.1 & 76.4 & 84.4 \\
\hline Yale University & 81.2 & 89.0 & 83.7 & 62.4 & 88.6 & - & 49.1 & 75.2 & 83.6 & 65.2 & 84.3 & 90.0 & 72.8 & 87.4 \\
\hline
\end{tabular}

Table 7: A representation of the HFSs associated with each university.

\begin{tabular}{lccccc} 
University & $\mathrm{A} \& \mathrm{H}$ & $\mathrm{L} \& \mathrm{M}$ & $\mathrm{ENG}$ & $\mathrm{SCI}$ & SOC \\
\hline Stanford University & $(0.868,0.871)$ & $(0.694,0.876,0.912)$ & $(0.919,0.921,0.933)$ & $(0.899,0.914,0.925)$ & $(0.801,0.892,0.936)$ \\
Harvard University & $(0.861,0.897)$ & $(0.913,0.982,1)$ & $(0.651,0.857)$ & $(0.902,0.923,1)$ & $(0.919,0.963,1)$ \\
University of Oxford & $(0.844,0.991)$ & $(0.609,0.911,0.923)$ & $(0.644,0.861,0.876)$ & $(0.723,0.873,0.904)$ & $(0.599,0.935,0.942)$ \\
University of Cambridge & $(0.839,0.935)$ & $(0.756,0.885,0.918)$ & $(0.748,0.888,0.905)$ & $(0.888,0.922,0.97)$ & $(0.594,0.875,0.912)$ \\
University of California, Berkeley & $(0.814,0.872)$ & $(0.58,0.816,0.856)$ & $(0.868,0.902,0.906)$ & $(0.899,0.934,0.963)$ & $(0.796,0.869,0.873)$ \\
Princeton University & $(0.812,0.865)$ & $(0.248,0.425,0.741)$ & $(0.711,0.816,0.895)$ & $(0.892,0.91,0.937)$ & $(0.764,0.844,0.911)$ \\
Yale University & $(0.812,0.89)$ & $(0.624,0.837,0.886)$ & $(0.491,0.752)$ & $(0.652,0.836,0.843)$ & $(0.728,0.874,0.9)$ \\
\hline
\end{tabular}

\subsection{Metarankings of universities: real exemplifications of our new ranking functions for HFSs}

The novel methodology in Section 3 can be used to provide metarankings of universities on the basis of the data in Tables 6 and 7 . The latter table provides the HFSs that characterize the respective universities (broadly speaking: cells represent HFEs and rows represent HFSs).

Our analyses are collected in Table 8, and Figure 2 provides a visual display of our conclusions. In both cases we report on rankings of the universities under inspection, obtained by the respective application of three 
ranking functions for HFEs: namely, the HFWs associated with the $\mathcal{S}_{1}, \mathcal{S}_{2}$ and $\mathcal{S}_{G M}$ scores.

We observe that there is full coincidence between the first and the third case, while the HFW obtained through $\mathcal{S}_{2}$ is significantly different.

Table 8: Rankings of the universities by applying the HFW respect to three focal scores.

\begin{tabular}{|c|c|c|c|c|c|c|}
\hline \multirow[b]{2}{*}{ University } & \multicolumn{2}{|c|}{$\mathcal{S}_{1}$} & \multicolumn{2}{|c|}{$\mathcal{S}_{2}$} & \multicolumn{2}{|c|}{$\mathcal{S}_{G M}$} \\
\hline & HFW & pos. & HFW & pos. & HFW & pos. \\
\hline Stanford University & 0.203 & 2 & 0.192 & 2 & 0.228 & 2 \\
\hline Harvard University & 0.507 & 1 & 0.445 & 1 & 0.506 & 1 \\
\hline University of Oxford & 0.040 & 6 & 0.106 & 4 & 0.038 & 6 \\
\hline University of Cambridge & 0.103 & 3 & 0.120 & 3 & 0.103 & 3 \\
\hline University of California, Berkeley & 0.084 & 4 & 0.082 & 5 & 0.066 & 4 \\
\hline Princeton University & 0.051 & 5 & 0.050 & 6 & 0.049 & 5 \\
\hline Yale University & 0.012 & 7 & 0.005 & 7 & 0.011 & 7 \\
\hline
\end{tabular}

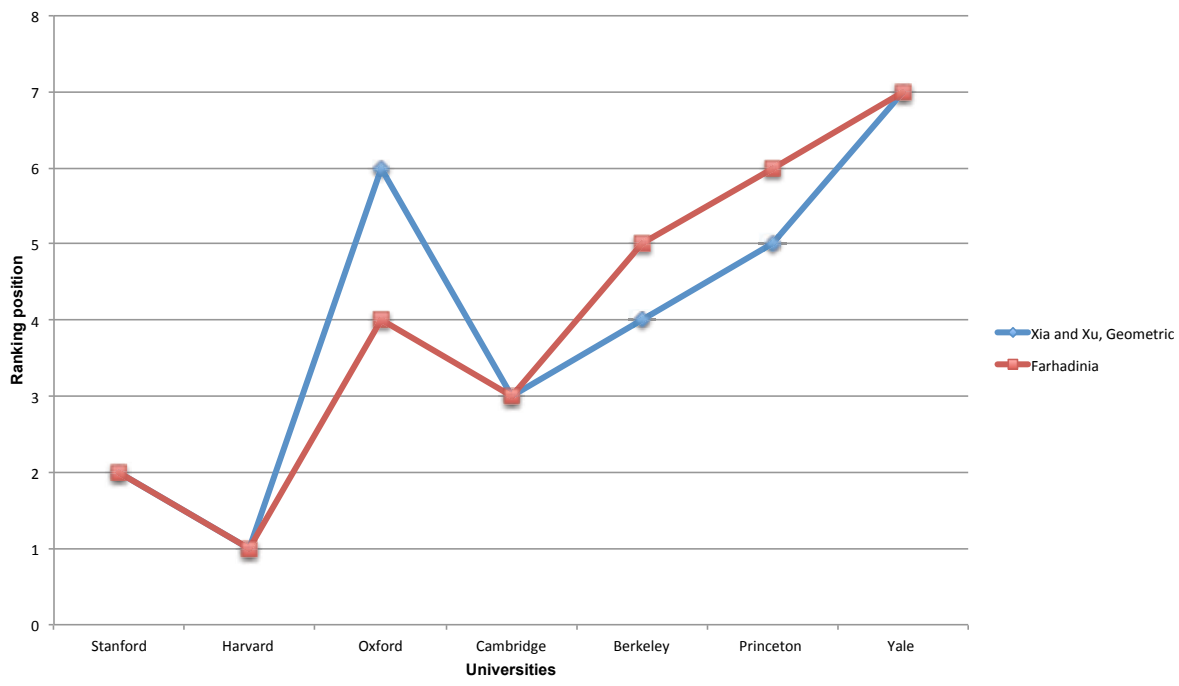

Figure 2: A graphical display of the ranking of the universities by the HFW procedure, as a function of three focal scores. 


\subsection{Comparison with other existing methodologies and discussion}

We proceed to perform a comparison of our proposal with relevant methodologies from the literature. To this purpose we divide the analysis to comply with the broad earlier approaches explained in Subsection 2.2.

\subsubsection{Ranking methodologies based on HFEs scores}

Table 9 collects the rankings obtained by four known methodologies on the data of Table 7 . Here we follow the approach suggested by Xia and $\mathrm{Xu}$ [7] and Xia et al. [17]. In order to rank HFSs, in [7, Section 4] the authors propose to use a $\mathrm{GHFWA}_{\lambda}$ operator (generalized hesitant fuzzy weighted averaging operator, which requires to fix a weight vector and depends on a $\lambda$ factor) in order to aggregate HFEs. But the same objective can be attained when we employ alternative aggregators or alternative scores on HFEs. ${ }^{3}$

In Table 9, the first column of digits captures the values by the ranking method that first applies the HFWA operator (hesitant fuzzy weighted averaging operator, which is GHFWA $_{\lambda}$ with $\lambda=1$ : cf., [7, Definition 9]) and then uses the $\mathcal{S}_{1}$ score. If we use the ranking method for HFSs that first applies the HFWA operator and then uses $\mathcal{S}_{2}$ instead, then we need to vary the computations above as in the second column of digits. We also present the corresponding indicators when we use the ranking method for HFSs that first applies the HFWG operator (or GHFWG ${ }_{\lambda}$ with $\lambda=1$ : cf., [7, Definition 10]) and then the $\mathcal{S}_{1}$ score, resp. the $\mathcal{S}_{2}$ score. To avoid controversies, we use equal weights throughout.

For the purpose of the comparison, observe that the aggregation by the HFWG operator with equal weights, followed by either Xia and Xu's or Farhadinia's score, produces the same ranking of the 7 universities. Similarly, the aggregation by the HFWA operator with equal weights, followed by these two scores, produces another common ranking of the 7 universities. The latter ranking coincides with that provided by the HFW associated with Farhadinia's score.

We summarize these results in Figure 3, which represents the conclusions in Table 9. Figure 3 compares with Figure 2, a representation of the conclusions in Table 8.

\footnotetext{
${ }^{3}$ Rodríguez et al. [9, Section 4] or Xu [8] report on many other alternative aggregators on HFEs, like GHFWG, GHFOWA or GHFOWG in [7] or QHFOWA, HFMOWA and HFMOWG in [17]. As to alternative scores on HFEs, Farhadinia's $\mathcal{S}_{2}$ or any other score mentioned in Subsection 2.2 can be employed.
} 
Table 9: Rankings of the universities by applying several aggregation operators.

\begin{tabular}{lcccccccc} 
& \multicolumn{3}{c}{ HFWG } & & \multicolumn{3}{c}{ HFWA } \\
\cline { 2 - 5 } \cline { 7 - 9 } University & $\mathcal{S}_{1}$ & $\mathcal{S}_{2}$ & pos. & & $\mathcal{S}_{1}$ & $\mathcal{S}_{2}$ & pos. \\
\hline Stanford University & 0.895 & 0.909 & 2 & & & 0.909 & 0.918 & 2 \\
Harvard University & 0.917 & 0.932 & 1 & & & 0.943 & 0.955 & 1 \\
University of Oxford & 0.829 & 0.864 & 5 & & 0.883 & 0.907 & 4 \\
University of Cambridge & 0.864 & 0.891 & 3 & & & 0.897 & 0.915 & 3 \\
University of California, Berkeley y & 0.846 & 0.865 & 4 & & 0.865 & 0.881 & 5 \\
Princeton University & 0.749 & 0.788 & 7 & & 0.838 & 0.853 & 6 \\
Yale University & 0.762 & 0.795 & 6 & & 0.807 & 0.829 & 7 \\
\hline
\end{tabular}

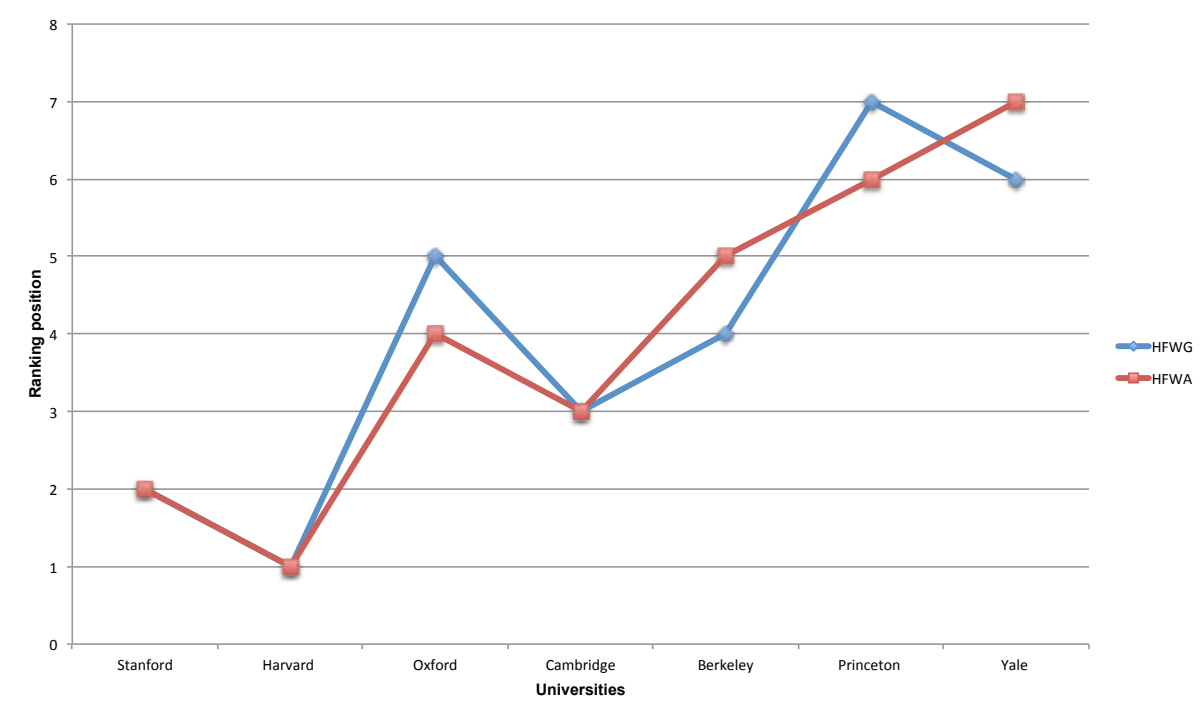

Figure 3: A graphical display of the ranking of the universities by two aggregation procedures. The subsequent application of Xia and Xu's or Farhadinia's score produces the same ranking in both instances.

In Table 10 we use some score functions on HFSs from Farhadinia [19] in order to provide rankings of the universities, as explained in subsection 2.2.1. In particular, we report on the analysis by six specifications: namely, when three respective scores are first applied on the raw data (namely, $\mathcal{S}_{1}$, $\mathcal{S}_{2}$ and $\mathcal{S}_{G M}$ ), and then either $\mathbb{S}_{A M}$ or $\mathbb{S}_{G M}$ (cf., [19, Definition 4.1]) is computed. We observe that the common ranking obtained in the second, fifth and sixth instances coincides with the ranking achieved by the application of the HFWG operator (cf., Table 9). 
A graphical display is given in Figure 4.

Table 10: Rankings of the universities by applying the arithmetic-mean $\mathbb{S}_{A M}$ and geometric-mean $\mathbb{S}_{G M}$ score functions to the $\mathcal{S}_{1}, \mathcal{S}_{2}$ and $\mathcal{S}_{G M}$ scores obtained for each subject.

\begin{tabular}{|c|c|c|c|c|c|c|c|c|c|c|c|c|}
\hline \multirow[b]{2}{*}{ University } & \multicolumn{4}{|c|}{$\mathcal{S}_{1}$} & \multicolumn{4}{|c|}{$\mathcal{S}_{2}$} & \multicolumn{3}{|c|}{$\mathcal{S}_{G M}$} & \multirow[b]{2}{*}{ pos. } \\
\hline & $\mathbb{S}_{A M}$ & pos. & $\mathbb{S}_{G M}$ & pos. & $\mathbb{S}_{A M}$ & post. & $\mathbb{S}_{G M}$ & pos. & $\mathbb{S}_{A M}$ & pos. & $\mathbb{S}_{G M}$ & \\
\hline Stanford University & 0.897 & 2 & 0.896 & 2 & 0.938 & 1 & 0.936 & 1 & 0.895 & 2 & 0.894 & 2 \\
\hline Harvard University & 0.921 & 1 & 0.919 & 1 & 0.912 & 2 & 0.911 & 2 & 0.920 & 1 & 0.917 & 1 \\
\hline University of Oxford & 0.839 & 5 & 0.838 & 5 & 0.876 & 4 & 0.875 & 4 & 0.828 & 5 & 0.826 & 5 \\
\hline University of Cambridge & 0.870 & 3 & 0.869 & 3 & 0.874 & 5 & 0.872 & 5 & 0.864 & 3 & 0.863 & 3 \\
\hline University of California, Berkeley & 0.851 & 4 & 0.849 & 4 & 0.902 & 3 & 0.902 & 3 & 0.848 & 4 & 0.846 & 4 \\
\hline Princeton University & 0.784 & 6 & 0.813 & 7 & 0.792 & 7 & 0.809 & 6 & 0.774 & 7 & 0.746 & 7 \\
\hline Yale University & 0.777 & 7 & 0.814 & 6 & 0.799 & 6 & 0.789 & 7 & 0.767 & 6 & 0.760 & 6 \\
\hline
\end{tabular}

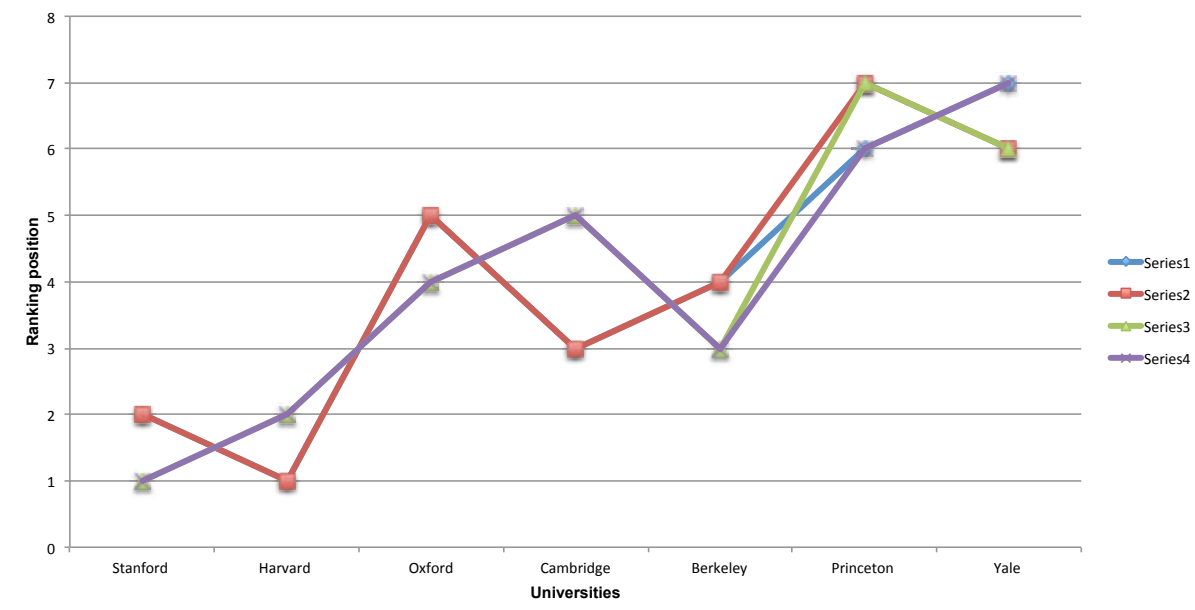

Figure 4: A graphical display of the ranking of the universities by Farahadinia's score functions (Table 10). Series 1 represents the ranking in the first column of the Table, Series 2 represents the common ranking obtained in the second, fifth and sixth columns of the Table, Series 3 represents the ranking in the third column of the Table and Series 4 represents the ranking in the fourth column of the Table.

\subsubsection{Ranking methodologies based on distance measures}

In Table 11 we obtain respective rankings of the universities by applying four methodologies from subsection 2.2.2: namely, distances to the ideal reference HFS by HWED, HWEHD, HWHD, and HWHHD respectively. We may observe that the verdict under HWHD coincides with the first specification of Farhadinia's score-based procedure (cf., Table 10), i.e., when $\mathcal{S}_{1}$ 
and then $\mathbb{S}_{A M}$ are applied. In addition, HWED provides the same ranking as the HFWG procedure (cf., Table 9) and three specifications of rankings suggested by scores on HFSs (cf., Table 10).

A graphical display is given in Figure 5.

Table 11: Rankings of the universities by applying four different distances to the ideal.

\begin{tabular}{lllllllll} 
University & HWED & pos. & HWEHD & pos. & HWHD & pos. & HWHHD & pos. \\
\hline Stanford University & 0.121 & 2 & 0.177 & 2 & 0.103 & 2 & 0.157 & 2 \\
Harvard University & 0.116 & 1 & 0.158 & 1 & 0.078 & 1 & 0.133 & 1 \\
University of Oxford & 0.211 & 5 & 0.346 & 5 & 0.160 & 5 & 0.330 & 6 \\
University of Cambridge & 0.166 & 3 & 0.261 & 4 & 0.130 & 3 & 0.236 & 4 \\
University of California, Berkeley & 0.175 & 4 & 0.243 & 3 & 0.149 & 4 & 0.225 & 3 \\
Princeton University & 0.290 & 7 & 0.388 & 7 & 0.216 & 6 & 0.310 & 5 \\
Yale University & 0.265 & 6 & 0.370 & 6 & 0.223 & 7 & 0.348 & 7 \\
\hline
\end{tabular}

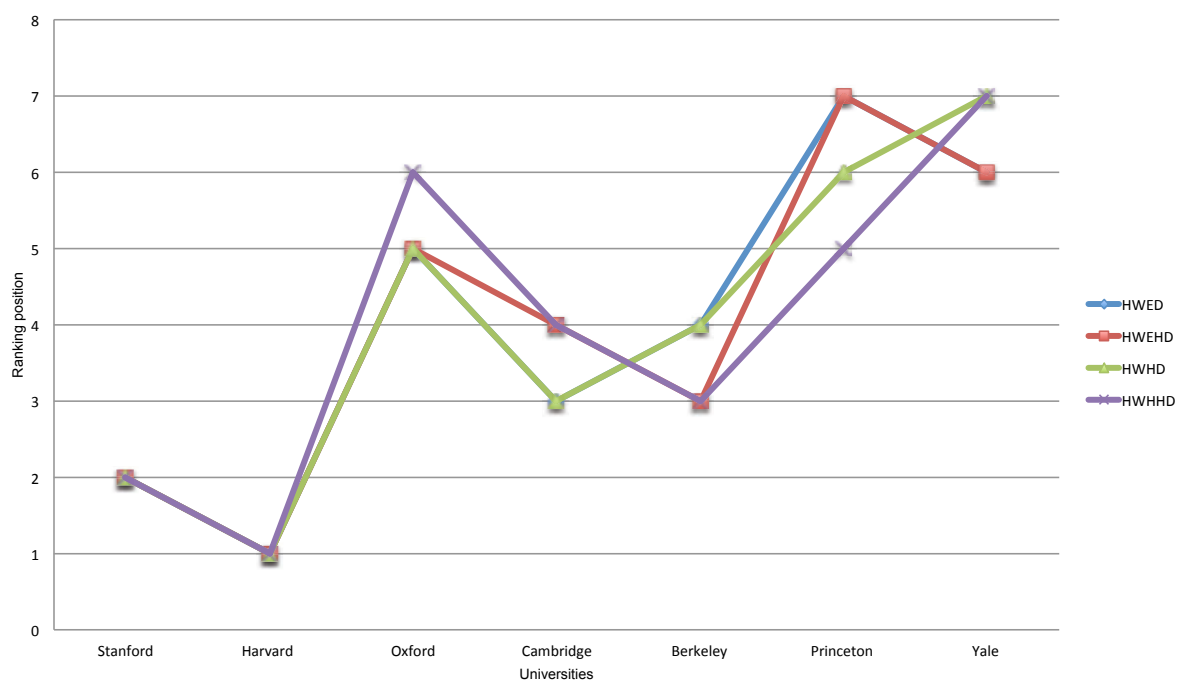

Figure 5: A graphical display of the ranking of the universities by four different distances to the ideal.

\section{Real application 2: a teacher performance assessment instru- ment}

In this Section we use our methodology to rank teachers' performance according to the results of their students' surveys. These surveys allow teachers to be more discerning about their own practice, and are often the only form 
of feedback on their teaching capabilities. Occasionally they are used for other practical purposes like hiring or compensations.

In order to submit our procedure, we rely on the real data in Table 12. It contains anonymized real data from student evaluations of staff of the department of Economics and Economic History, Universidad de Salamanca, Spain. In this University students are asked to provide feedback on specific aspects of their classroom experiences on a biyearly basis. For each subject and teacher, students submit their level of agreement (0 means "strongly disagree", 5 means "fully agree") to statements such as "He/she explains with clarity", "He/she clearly answers the questions raised in the classroom", "Our activities and duties are well organized and structured", or "Overall, my degree of satisfaction with him/her has been good". All steps are taken to protect students confidentiality. These surveys are then aggregated by subjects and confidentially submitted to the evaluated person. Figure 6 depicts an anonymized part of a subject report received by one evaluated person, where the first column of digits is the only individual part of the report (the other columns provided for comparison: average of the Department and of the Degree). Hence the staff in the Department receive a number of records that depends on their teaching allocations. Table 12 has one row for each evaluated person in our sample, one column for each of the eleven fields in the survey (attributes), and various assessments for each person that vary with his/her teaching allocation. We have normalized these figures to the interval $[0,1]$ by dividing them by 5 . These normalized assessments are already collected as HFSs for brevity, since we arrive at this form as in the real example of Section 5. We can now apply our methodology to Table 12 in order to rank the staff by their teaching performance during the academic year 2013-14. Our analyses are collected in Table 13.

A comparative study could be performed as in Section 5, but we skip it for brevity. Let us just mention that despite the case of the example in Section 5 (cf., Table 8 and Figure 1), Table 13 proves that the HFWs associated with the three focal scores we have been using convey distinct rankings. 
DATOS DE CONTEXTO

Media de los estudiantes que responden la encuesta:

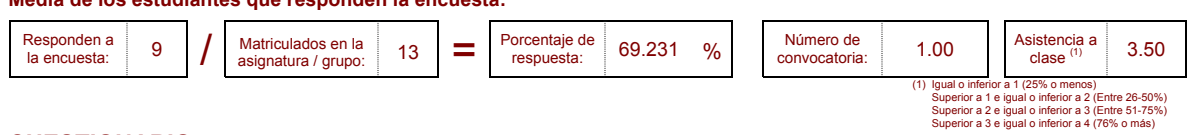

CUESTIONARIO

\begin{tabular}{|c|c|c|c|c|c|}
\hline \multicolumn{2}{|r|}{ Valoraciones medias de las respuestas de los estudiantes ${ }^{(2)}$ : } & \multirow{2}{*}{$\begin{array}{l}\begin{array}{c}\text { Media del } \\
\text { Profesor }\end{array} \\
4.11\end{array}$} & \multirow{2}{*}{\begin{tabular}{|c|}
$\begin{array}{c}\text { No } \\
\text { procede }^{(3)}\end{array}$ \\
0 \\
0
\end{tabular}} & \multirow{2}{*}{\begin{tabular}{|c|}
$\begin{array}{c}\text { Media } \\
\text { Departamento }^{(4)}\end{array}$ \\
3.61
\end{tabular}} & \multirow{2}{*}{$\begin{array}{c}\frac{c_{\text {Media }}{ }^{(4)}}{\text { Titulación }^{(4)}} \\
4.36\end{array}$} \\
\hline 1 & El/la profesor/a explica con claridad. & & & & \\
\hline 2 & $\begin{array}{l}\text { Resuelve las dudas plateadas y orienta a los estudiantes en el desarrollo de } \\
\text { sus tareas. }\end{array}$ & 4.00 & 0 & 3.65 & 4.33 \\
\hline 3 & $\begin{array}{l}\text { Organiza y estructura bien las actividades o tareas que realizamos con el/la } \\
\text { profesor/a (aula, laboratorio, taller, seminario, trabajo de campo, etc.). }\end{array}$ & 3.89 & 0 & 3.59 & 4.23 \\
\hline 4 & $\begin{array}{l}\text { Las actividades o tareas (teóricas, prácticas, de trabajo individual, en grupo, } \\
\text { etc.) son provechosas para lograr los objetivos de la asignatura. }\end{array}$ & 4.00 & 0 & 3.53 & 4.19 \\
\hline 5 & $\begin{array}{l}\text { Favorece la participación del estudiante en el desarrollo de la actividad } \\
\text { docente. }\end{array}$ & 3.56 & 0 & 3.50 & 4.25 \\
\hline 6 & $\begin{array}{l}\text { Está accesible para ser consultado por los estudiantes (tutorías, orientación } \\
\text { académica, ...) }\end{array}$ & 4.22 & 0 & 3.77 & 4.30 \\
\hline 7 & $\begin{array}{l}\text { Ha facilitado mi aprendizaje y gracias a su ayuda he logrado mejorar mis } \\
\text { conocimientos, habilidades o destrezas. }\end{array}$ & 3.78 & 0 & 3.35 & 4.16 \\
\hline 8 & $\begin{array}{l}\text { Los recursos didácticos utilizados por el/la profesor/a son adecuados para } \\
\text { facilitar el aprendizaje. }\end{array}$ & 3.67 & 0 & 3.47 & 4.14 \\
\hline 9 & $\begin{array}{l}\text { La bibliografía y los materiales didácticos facilitados son útiles para realizar } \\
\text { las tareas y para el aprendizaje. }\end{array}$ & 4.00 & 0 & 3.47 & 4.20 \\
\hline 10 & $\begin{array}{l}\text { Los métodos de evaluación se corresponden con el desarrollo docente de la } \\
\text { materia (responder sólo en caso de que se hayan realizado pruebas de } \\
\text { evaluación de la asignatura). }\end{array}$ & 4.00 & 0 & 3.55 & 4.22 \\
\hline 11 & Mi grado de satisfacción general con el/la profesor/a ha sido bueno. & 4.11 & 0 & 3.55 & 4.32 \\
\hline $\begin{array}{l}\text { (2) } 1 \\
4 \\
\text { (3) } \operatorname{lin} \\
\text { la } \\
\text { (4) }\end{array}$ & 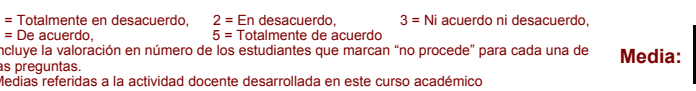 & 3.94 & & 3.55 & 4.24 \\
\hline
\end{tabular}

Unidad de Evaluación de la Calidad - C.M.San Bartolomé - Plaza Fray Luis de León 1, 37008 Salamanca - E-mail: qualitas@ usal.es - Web: http://qualitas.usal.es - Tel./Fax: (34) 923294638

Figure 6: Anonymized part of a subject report, Universidad de Salamanca (in Spanish).

\section{Conclusion}

In this paper we give a novel methodology for ranking hesitant fuzzy sets. It introduces a pioneering bridge between this topic and Social Choice.

As to methodological differences with existing procedures, there is one that deserves explicit discussion. We refer to the analysis of the metarankings of universities for convenience of explanation. The indicators in Table 9 are computed one by one, i.e., they are attached with the corresponding alternative or HFS independently of the structure of the other HFSs in the problem. However the indicators in Table 8 must be computed globally since they build on a matrix that depends on comparisons among related 
Table 12: A representation of the HFSs associated with each staff member.

\begin{tabular}{|c|c|c|c|c|c|c|}
\hline \multirow[b]{2}{*}{ Staff } & \multicolumn{6}{|c|}{ Criteria } \\
\hline & 1 & 2 & 3 & 4 & 5 & 6 \\
\hline 1 & $(0.474,0.822)$ & $(0.566,0.8)$ & $(0.558,0.778)$ & $(0.6,0.8)$ & $(0.434,0.712)$ & $(0.722,0.844)$ \\
\hline 2 & $(0.77,0.8,0.83)$ & $(0.824,0.838,0.848)$ & $(0.636,0.68,0.772)$ & $(0.71,0.75,0.752)$ & $(0.752,0.788,0.806)$ & $(0.676,0.716,0.766)$ \\
\hline 3 & $(0.71,0.73,0.784,0.872)$ & $(0.772,0.8,0.856,0.896)$ & $(0.678,0.728,0.734,0.768)$ & $(0.672,0.72,0.73,0.734)$ & $(0.754,0.766,0.808,0.81)$ & $(0.64,0.746,0.756,0.77)$ \\
\hline 4 & $(0.77,0.83)$ & $\begin{array}{l}(0.764,0.834) \\
(0.00,0.090)\end{array}$ & 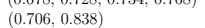 & $\begin{array}{l}(0.702,0.818) \\
(0.0,0.704)\end{array}$ & $(0.764,0.856)$ & $(0.77,0.85)$ \\
\hline 5 & $(0.382,0.662)$ & $(0.522,0.698)$ & $(0.634,0.7)$ & $(0.582,0.752)$ & $(0.556,0.658)$ & $(0.66,0.722)$ \\
\hline 6 & $(0.418,0.566)$ & $(0.47,0.634)$ & $(0.426,0.566)$ & $(0.486,0.662)$ & $(0.418,0.656)$ & $(0.47,0.6)$ \\
\hline 7 & $(0.476,0.576,0.606,0.73)$ & $(0.65,0.666,0.69,0.746)$ & $(0.446,0.48,0.488,0.64)$ & $(0.438,0.52,0.526,0.6,0.65)$ & $(0.64,0.662,0.7)$ & $(0.49,0.658,0.662,0.746)$ \\
\hline 8 & $(0.65,0.738,0.8,0.876)$ & $(0.65,0.754,0.8,0.842)$ & $(0.55,0.584,0.6,0.642)$ & $\begin{array}{l}(0.6,0.63,0.7) \\
\text { (n) }\end{array}$ & $(0.75,0.816,0.866)$ & $(0.65,0.7,0.708,0.85)$ \\
\hline 9 & $(0.672,0.748,0.76)$ & $(0.696,0.748,0.808)$ & $(0.45,0.512,0.632)$ & $(0.528,0.63,0.664)$ & $(0.6,0.762,0.784)$ & $(0.456,0.756,0.792)$ \\
\hline \multirow[t]{2}{*}{10} & $(0.726,0.832)$ & $(0.726,0.79)$ & $(0.586,0.664)$ & $(0.534,0.664)$ & $(0.66,0.758)$ & $(0.762,0.844)$ \\
\hline & \multicolumn{6}{|c|}{ Criteria } \\
\hline Staff & 7 & 8 & 9 & 10 & 11 & \\
\hline 1 & $(0.456,0.756)$ & $(0.548,0.734)$ & $(0.6,0.8)$ & $(0.588,0.8)$ & $(0.494,0.822)$ & \\
\hline 2 & $(0.782,0.804,0.82)$ & $(0.624,0.648,0.652)$ & $(0.834,0.858,0.86)$ & $(0.62,0.636,0.704)$ & $(0.812,0.854,0.896)$ & \\
\hline 3 & $(0.754,0.808,0.81,0.872)$ & $(0.644,0.672,0.676,0.688)$ & $(0.754,0.8,0.858,0.904)$ & $(0.59,0.606,0.608,0.682)$ & $(0.826,0.828,0.902,0.944)$ & \\
\hline 4 & $(0.676,0.8)$ & $(0.708,0.86)$ & $\begin{array}{l}(0.716,0.846) \\
(0.00,0)\end{array}$ & $(0.702,0.834)$ & $(0.724,0.858)$ & \\
\hline 5 & $(0.454,0.674)$ & $(0.548,0.732)$ & $(0.556,0.74)$ & $(0.592,0.76)$ & $(0.446,0.658)$ & \\
\hline 6 & $(0.418,0.6)$ & $(0.574,0.656)$ & $(0.574,0.662)$ & $(0.558,0.634)$ & $(0.418,0.656)$ & \\
\hline 7 & $(0.66,0.73,0.786,0.794)$ & $(0.566,0.576,0.594,0.63)$ & $(0.626,0.63,0.668,0.704)$ & $(0.534,0.538,0.6)$ & $(0.45,0.5,0.574,0.642)$ & \\
\hline 8 & $(0.75,0.75,0.784,0.892)$ & $(0.5,0.65,0.726,0.77)$ & $(0.5,0.65,0.754,0.816)$ & $(0.554,0.6,0.642)$ & $(0.65,0.676,0.8,0.816)$ & \\
\hline 9 & $(0.752,0.776,0.8)$ & $(0.616,0.652,0.736)$ & $(0.6,0.72,0.762)$ & $(0.57,0.6,0.64)$ & $(0.536,0.652,0.712)$ & \\
\hline 10 & $(0.718,0.8)$ & $(0.61,0.66)$ & $(0.674,0.684)$ & $(0.484,0.518)$ & $(0.712,0.716)$ & \\
\hline
\end{tabular}

Table 13: Rankings of staff by teaching performance, academic year 2013-14, according to their respective HFWs.

\begin{tabular}{lllllllll} 
& \multicolumn{2}{c}{ Xia and Xu } & & \multicolumn{2}{c}{ Farhadinia } & & \multicolumn{2}{c}{ Geometric } \\
\cline { 2 - 3 } Staff & HFW & pos. & & HFW & pos. & & HFW & pos. \\
\hline Number 1 & 0.0344308 & 6 & & 0.000493097 & 6 & & 0.0329902 & 5 \\
Number 2 & 0.196536 & 3 & & 0.730558 & 1 & & 0.177189 & 3 \\
Number 3 & 0.203587 & 2 & & 0.00764202 & 3 & & 0.217913 & 2 \\
Number 4 & 0.423015 & 1 & & 0.00251855 & 4 & & 0.444753 & 1 \\
Number 5 & 0.0136762 & 8 & & 0.000180212 & 8 & & 0.0112894 & 8 \\
Number 6 & 0.00141894 & 10 & & 0.000005168 & 10 & & 0.00212334 & 10 \\
Number 7 & 0.00953274 & 9 & & 0.0000539497 & 9 & & 0.00448744 & 9 \\
Number 8 & 0.0485923 & 4 & & 0.000884115 & 5 & & 0.0488669 & 4 \\
Number 9 & 0.0341514 & 7 & & 0.257395 & 2 & & 0.029482 & 7 \\
Number 10 & 0.0350599 & 5 & & 0.000270001 & 7 & & 0.0309057 & 6 \\
\hline
\end{tabular}

scores. Borrowing from Herrero [24]: the reason is that they attempt to capture "the relative support of the alternatives (i.e., how important one alternative is with respect to another one, taking the full set of alternatives into account)". Admittedly this feature may seem a handicap with respect to previous methodologies. But the aspect that we gain is a novel interaction with a separated branch of the literature, which can be beneficial for future investigations.

With respect to computational complexity, there are two steps to analyze: 
the calculation of $C$ and then the calculation of a normalized eigenvector for its known dominant eigenvalue. Let us insist that the $C$ matrix is very simple to produce (it requires to attach scores and then compare their values). And nowadays plenty of tools permit to find the eigenvectors associated with a known eigenvalue. Ultimately, this problem boils down to a known system of $k$ linear equations, which can be solved very efficiently e.g., by Gauss' algorithm.

Two applications to real problems are put forward that validate our methodology. Metarankings of universities are derived on updated evaluations made by three agencies, and a teacher performance assessment instrument is developed and exemplified with real data. By doing so we provide new justification of the theoretical model by HFSs, and of the need for continuing studying ranking functions for HFSs.

\section{Acknowledgment}

The authors thank the anonymous reviewers and José Salmerón (handling editor) for their valuable comments and recommendations. The authors are grateful to Prof. Zeshui Xu for helpful suggestions and encouragement. Financial support by the Spanish Ministerio de Ciencia e Innovación under Project ECO2012-31933 (J. C. R. Alcantud), Project ECO2012-32178, Project CGL2008-06003-C03-03/CLI (R. de Andrés Calle), and Junta de Andalucía Project P09-SEJ-05404 (M. J. M. Torrecillas) is gratefully acknowledged.

\section{References}

[1] R. Montes, A. Sánchez, P. Villar, F. Herrera, A web tool to support decision making in the housing market using hesitant fuzzy linguistic term sets, Applied Soft Computing (Forthcoming).

[2] T. Tanino, Fuzzy preference orderings in group decision making, Fuzzy Sets and Systems 12(2) (1984) 117-131.

[3] J. Fodor, M. Roubens, Fuzzy preference modelling and multicriteria decision support, Kluwer Academic Publishers, 1994.

[4] J. Kacprzyk, M. Fedrizzi, H. Nurmi, Consensus under fuzziness, Kluwer Academic Publishers, 1997. 
[5] A. Mardani, A. Jusoh, E. K. Zavadskas, Fuzzy multiple criteria decisionmaking techniques and applications two decades review from 1994 to 2014, Expert Systems with Applications 42 (8) (2015) 4126-4148.

[6] V. Torra, Hesitant fuzzy sets, International Journal of Intelligent Systems 25(6) (2010) 529-539.

[7] M. Xia, Z. Xu, Hesitant fuzzy information aggregation in decision making, International Journal of Approximate Reasoning 52 (2011) 395-407.

[8] Z. Xu, Hesitant Fuzzy Sets Theory, Vol. 314 of Studies in Fuzziness and Soft Computing, Springer International Publishing, 2014.

[9] R. Rodríguez, L. Martínez, V. Torra, Z. Xu, F. Herrera, Hesitant fuzzy sets: state of the art and future directions, International Journal of Intelligent Systems 29 (2014) 495-524.

[10] B. Farhadinia, A novel method of ranking hesitant fuzzy values for multiple attribute decision-making problems, International Journal of Intelligent Systems 28 (2013) 752-767.

[11] X. Zhou, Q. Li, Multiple attribute decision making based on hesitant fuzzy einstein geometric aggregation operators, Journal of Applied Mathematics (2014) Article ID 745617.

[12] Z. Xu, M. Xia, On distance and correlation measures of hesitant fuzzy information, International Journal of Intelligent Systems 26 (2011) 410425 .

[13] H. Liao, Z. Xu, Satisfaction degree based interactive decision making under hesitant fuzzy environment with incomplete weights, International Journal of Uncertainty, Fuzziness and Knowledge-Based Systems 22 (04) (2014) 553-572.

[14] X. Zhang, Z. Xu, Hesitant fuzzy QUALIFLEX approach with a signed distance-based comparison method for multiple criteria decision analysis, Expert Systems with Applications 42 (2) (2015) 873 - 884.

[15] L. Zhou, J. Wu, H. Chen, Linguistic continuous ordered weighted distance measure and its application to multiple attributes group decision making, Applied Soft Computing 25 (2014) 266 -276. 
[16] X. Zhang, Z. Xu, The TODIM analysis approach based on novel measured functions under hesitant fuzzy environment, Knowledge-Based Systems 61 (0) (2014) $48-58$.

[17] M. Xia, Z. Xu, N. Chen, Some hesitant fuzzy aggregation operators with their application in group decision making, Group Decision and Negotiation 22 (2013) 259-279.

[18] C. Tan, W. Yi, X. Chen, Hesitant fuzzy hamacher aggregation operators for multicriteria decision making, Applied Soft Computing 26 (2015) $325-349$.

[19] B. Farhadinia, A series of score functions for hesitant fuzzy sets, Information Sciences 277 (2014) 102-110.

[20] R. Yager, On choosing berween fuzzy subsets, Kybernete 9 (1980) 151154.

[21] R. Yager, A procedure for ordering fuzzy subsets of the unit interval, Information Sciences 24 (1981) 143-161.

[22] G. Bortolan, R. Degani, A review of some methods for ranking fuzzy subsets, Fuzzy Sets and Systems 15 (1985) 1-19.

[23] J. C. R. Alcantud, R. de Andrés Calle, T. González-Arteaga, A new health classification scheme based on fuzzy hesitant information, in: J. M. Alonso, H. Bustince, M. Reformat (Eds.), Proceedings of the 2015 Conference of the International Fuzzy Systems Association and the European Society for Fuzzy Logic and Technology, Atlantis Press, 2015, pp. $1245-1252$.

[24] C. Herrero, An endogenous scoring rule, Mimeo.

[25] J. Alcantud, Fuzzy soft set based decision making: a novel alternative approach, Mimeo.

[26] J. Peng, J. Wang, J. Wang, L. Yang, X. Chen, An extension of ELECTRE to multi-criteria decision-making problems with multi-hesitant fuzzy sets, Information Sciences 307 (2015) 113-126. 
[27] X. Zhang, Z. Xu, Hesitant fuzzy QUALIFLEX approach with a signed distance-based comparison method for multiple criteria decision analysis, Expert Systems with Applications 42 (2) (2015) 873-884.

[28] Z. Xu, X. Zhang, Hesitant fuzzy multi-attribute decision making based on TOPSIS with incomplete weight information, Knowledge-Based Systems 52 (2013) 53-64.

[29] N. Zhang, G. Wei, Extension of VIKOR method for decision making problem based on hesitant fuzzy set, Applied Mathematical Modelling 37 (7) (2013) 4938-4947.

[30] Z. Xu, M. Xia, Distance and similarity measures for hesitant fuzzy sets, Information Sciences 181 (2011) 2128-2138.

[31] D. Li, W. Zeng, J. Li, New distance and similarity measures on hesitant fuzzy sets and their applications in multiple criteria decision making, Engineering Applications of Artificial Intelligence 40 (2015) 11-16.

[32] M. Xia, Z. Xu, B. Zhu, Geometric Bonferroni means with their application in multi-criteria decision making, Knowledge-Based Systems 40 (0) (2013) 88-100.

[33] G. Wei, Hesitant fuzzy prioritized operators and their application to multiple attribute decision making, Knowledge-Based Systems 31 (2012) 176-182.

[34] J. J. Liou, C.-Y. Tsai, R.-H. Lin, G.-H. Tzeng, A modified VIKOR multiple-criteria decision method for improving domestic airlines service quality, Journal of Air Transport Management 17 (2) (2011) 57 - 61.

[35] ShanghaiRanking Consultancy, Academic ranking of world universities (ARWU), Available in: http://www.shanghairanking.com/ (2014).

[36] Quacquarelli Symonds Limited, QS world university rankings, Available in: http://www.topuniversities.com/university-rankings (2014).

[37] Thomson Reuters, Times higher education world university rankings 2013-2014, Available in: http://www.timeshighereducation.co.uk/world-university-rankings/ (2014). 